\title{
Assessing Warning Signs of Relapse in Drug Addicts: Translation and Validation of Advance Warning for Relapse (AWARE) Questionnaire
}

\author{
NAMOOD-E-SAHAR ${ }^{1}$, IRUM NAQVI ${ }^{2}$ \\ ${ }^{1}$ Research Assistant, National Institute of Psychology, Quaid-i-Azam University, Islamabad, Pakistan \\ ${ }^{2}$ Assistant Professor, National Institute of Psychology, Quaid-i-Azam University, Islamabad, Pakistan \\ Correspondence to Namood-e-Sahar, E-mail: namoodmphil17@nip.edu.pk, Telephone: +92 3030597021
}

\begin{abstract}
Aim: To translate and validate Advance Warning of Relapse (AWARE) Questionnaire in to Urdu language for assessing relapse among drug addicts of Pakistan.

Methods: The research comprises of translation of AWARE, temporal validation of translated scale, and its construct validation. Two items $(22,28)$ were modified keeping structural and functional equivalence. Sample for validation was obtained from rehabilitation centers of Islamabad and Rawalpindi, with age ranging from $18-60$ years $(M=29.3 ; S D=8.2)$. Temporal validity was determined by test-retest reliability. Exploratory factor analysis (EFA) was used to determine the valid factor structure, whose model fitness was assessed using Confirmatory factor analysis (CFA). Reliability was used to assess the internal consistency.

Results: It was found that translated scale possess significant temporal validity $(\alpha=.81)$. EFA demonstrate a unifactorial structure $(\lambda>.30)$ explaining variance of about $34.3 \%$; such that four items $(8,14,20,26)$ were removed based on poor factor loadings. Indices of model fit through CFA confirm factor structure. Reliability of scale was also significant $(\alpha=.90)$.

Conclusion: Findings suggested the reliable and valid nature of AWARE-Urdu to assess warning signs of relapse in drug addicts.
\end{abstract}

Keywords: Warning signs, relapse, drug addicts, Urdu translation, validation, AWARE

\section{INTRODUCTION}

Addiction is a recurring and difficult to control behavior that is signified by physical and psychological inability to avoid a substance or activity despite of its prevailing harmful effect. ${ }^{1}$ Drug addiction however is defined by National Institute on Drug Abuse (NIDA) as the chronic disease involving the behavior of drug seeking and usage which is compulsive in nature; hence long lasting and is excessively repeated despite harmful consequences for the individual ${ }^{2}$. It is a menace to the society as it results in enslaving the individual of drug or substance ${ }^{3}$ and the addicted person start deteriorating physically by being at high risk of HIV, lung or heart diseases, cancer ${ }^{4,5}$, mentally ${ }^{6}$, emotionally ${ }^{7}$ and socially 8,9 .

Drug addiction is associated with the intense compulsive urges to use the drug and thus there are high chances of relapse 10; that is a return to addictive behavior signified by recurrence of past condition that exist before treatment ${ }^{11}$. These urges are caused by biological changes ${ }^{12}$ as well as the psychological and social determinants ${ }^{13,14}$.

Relapse is thus very difficult to fight with because the individual has to again overcome effectively all the biological as well as the psychosocial factors which are associated with the relapse condition. Drug relapse prevention is so a very significant part of the recovery for addicts because of the persisting risk of relapse for many years after the treatment. ${ }^{2}$ Statistics indicate that in Pakistan approximately 6.7 million people are drug addicts comprising of $6 \%$ of the total population ${ }^{15}$ and of which around $70 \%$ are with history of relapse ${ }^{16}$. This indicates an alarming situation and thus a dire need to study the concept of relapse among drug addicts of Pakistan. This objective could be achieved by using a reliable and valid measure to assess the relapse.

Different scales have been developed to estimate the relapse; of them Stimulant Relapse Risk Scale (SSRS) estimates the risk for relapse ${ }^{17}$, Time to Relapse Questionnaire (TRQ) determine the time taken by the individual from thought of initial drug to the actual use of drug ${ }^{18}$. Advance Warning of Relapse (AWARE) Questionnaire is a valid measure in this regard with good predictability for the occurrence of relapse $(r=.42, p<.001)^{19}$. The measure is also used in the recent researches which provides evidence for the applicability of the measure in assessing relapse $\mathrm{e}^{20,21}$.

Received on 17-04-2021

Accepted on 19-08-2021
The Advance Warning of Relapse Questionnaire was devised with an intent to assess chances of relapse by determining the warning signs associated with relapse condition, as described by Gorski and Miller22. These warning signs can be broken down into three main categories; i.e., emotional, mental, and physical relapse. During the "emotional type of relapse the person although not consciously thinking about drug usage but they are setting themselves up for it and are in some denial about the possibility that a relapse can occur. The second category is "mental relapse" in which the person is thinking about drug usage, but they are at the state of war with themselves between wish of using the drug and not using it. This state of inner war makes the person tired and less able to deal effectively with the situation. The last one is "physical relapse" when a person finally returns to drug use $\mathrm{e}^{23}$.

Urdu translated or adapted version of the scale is not yet devised. The translation and validation of research instruments is however of great significance for addressing cultural variability and establishing the cultural equivalence ${ }^{24}$. The establishment of construct validity and psychometrics of the research instruments is also very important so as to ensure factor structures around the culture the psychometrically sound data; that is reliable and valid. ${ }^{25}$ The present study thus aimed to translate, modify, and validate the scale thus making it culturally adaptable for drug addicts.

\section{METHOD}

Research Type: The study was conducted to obtain a valid and reliable Urdu version of Advance Warning of Relapse (AWARE) Questionnaire after approval from IRB. The data was collected between November 2018 to March 2019. The population of the study comprised of drug addicts who are currently under treatment. Sample $(N=165)$ was approached at the rehabilitation centers Rawalpindi and Islamabad.

Data Collection Tools: The data collection was carried out through AWARE Questionnaire.

Advance Warning of Relapse Questionnaire: This is a selfreport measure designed to assess the warning signs associated with relapse, as described by Gorski. ${ }^{22}$ The current version of the scale comprised of 28 -items and was refined from original version of 37-items after subsequent analyses. The items are scored on 17 Likert type rating scale; such that, 1 denotes never and 7 denotes always. The scoring is reversed for the five items of the questionnaire: $8,14,20,24,26$. The higher score indicate prevalence of more warning signs of relapse and thus lower 
prevention against relapse. The score ranges from lowest possible score of 28 to highest possible score of 196 . The scale possesses good internal consistency $(\alpha=.92)$ as well as test-retest reliability $(\alpha=.92)^{26}$.

Data Collection: Data was collected by administering the questionnaire in an individual setting with drug addicts.

Data Analysis: The data was analyzed by using test-retest reliability to establish temporal validity. Cronbach's $\alpha$ reliability assessed the internal consistency of the questionnaire. Factor analysis was used to determine factor structure (i.e., exploratory factor analysis) and confirm that factor structure (i.e., confirmatory factor analysis).

Translation of Advance Warning of Relapse Questionnaire: The original author of Advance Warning of Relapse (AWARE) Questionnaire "William R. Miller" was contacted via E-mail to acquire permission for translation and modification. The translation was carried out by using the well-established method of forwardback translation ${ }^{27}$.

Validity and Reliability of Advance Warning of Relapse Questionnaire: The validity of questionnaire was assessed by determining linguistic, temporal, and construct validity. The reliability was assessed by calculating the internal consistency.

Linguistic Validity: The independent Urdu translations were obtained from five bilingual experts and these forward translations were reviewed by committee of subject matter experts. The finalized Urdu version of the scale was then back translated into English by five bilinguals and the appropriate final back translation was selected through committee approach.

Temporal Validity: Temporal validation was established by determining the consistency of scores across the time through testretest reliability. The purpose was achieved by administering the Urdu version of Advance Warning of Relapse (AWARE) Questionnaire "AWARE-Urdu" on a sample of 25 drug addicts. The sample $(N=25)$ was obtained from a rehabilitation center in vicinity of Islamabad which comprised of the participants aged 18-42 years $(M=28.4, S D=5.9)$, majority had secondary level of education, and all were males. Prior to the administration permission was acquired from the authorities of rehabilitation centers by briefing them about the purpose and that the participants were to be re-tested after the time period of one-week. Urdu-Urdu test-retest condition was used to accomplish the goal of study. The Urdu translated version of instrument was administered on the participants and after oneweek same participants were re-tested using same Urdu version of the instrument.

Construct Validity: The factor structures for construct validity were determined through EFA and confirmed by using CFA. Independent samples were obtained for both analyses comprising of $140(M=30.03 ; S D=8.35)$ and $150(M=29.45 ; S D=8.83)$ drug addicts respectively from drug rehabilitation centers in the locality of Islamabad and Rawalpindi. Authorities were assured about all the ethical consideration including; confidentiality, anonymity, and psychological safety of the participants.

Internal Consistency: Internal consistency was determined by using the reliability estimates.

Ethical Principles of the Study: The permission for the translation and modification for AWARE was obtained from original author. The author was ensured of the ethical considerations and also of maintaining the functional and conceptual equivalence. The study participants for validation of instrument were ensured about the anonymity and confidentiality of their responses.

\section{RESULTS}

The results for validation and psychometric properties of the translated instrument are mentioned as follows:

Content Validity: The content validity was achieved by the committee of experts and the final back translation was sent to the original author of AWARE for the review. The author mentioned suggestion regarding the conceptual equivalence based on which Item-1 was changed from "I feel anxious or uncertain about my ability to quit drug" to "I feel anxious or uncertain about my ability to stay drug free" (Table 1).

Table 1: The Modified Item of AWARE-Urdu Based on Author's Review

\begin{tabular}{|c|c|}
\hline Previous item before review & Modified item after review \\
\hline 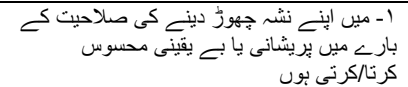 & 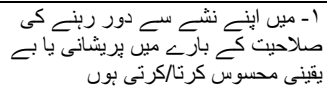 \\
\hline
\end{tabular}

Table 2. Factor loadings for Advance Warning of Relapse QuestionnaireUrdu through Principal Component Analysis ( $N=140)$

\begin{tabular}{|c|c|c|c|c|c|}
\hline \multirow[t]{2}{*}{ Items } & \multicolumn{2}{|c|}{ Components } & \multirow[t]{2}{*}{ Items } & \multicolumn{2}{|c|}{ Components } \\
\hline & $\lambda$ & $h^{2}$ & & $\lambda$ & $h^{2}$ \\
\hline 11. & .71 & .57 & 15. & .62 & .45 \\
\hline 10. & .70 & .49 & 17. & .61 & .38 \\
\hline 19. & .70 & .47 & 9. & .58 & .35 \\
\hline 22. & .69 & .45 & 23. & .57 & .31 \\
\hline 13. & .67 & .48 & 2. & .55 & .30 \\
\hline 6. & .67 & .45 & 1. & .53 & .30 \\
\hline 3. & .67 & .45 & 28. & .52 & .31 \\
\hline 4. & .66 & .45 & 16. & .50 & .24 \\
\hline 7. & .65 & .43 & 21. & .40 & .19 \\
\hline 12. & .64 & .38 & 5. & .32 & .20 \\
\hline 25. & .62 & .38 & 18. & .31 & .14 \\
\hline 27. & .62 & .41 & 24. & .30 & .18 \\
\hline \multicolumn{5}{|c|}{ Eigen Values } & 8.24 \\
\hline \multicolumn{5}{|c|}{$\%$ of Variance } & 34.33 \\
\hline
\end{tabular}

Figure 1. Measurement model of Advance Warning of Relapse Questionnaire-Urdu (24 items).

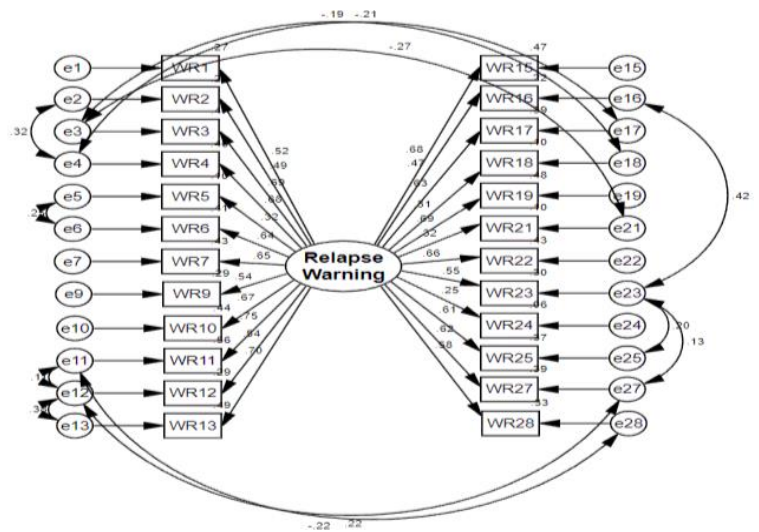

Temporal Validity: Temporal validity was established by assessing the test-retest reliability of the translated version of scale which demonstrated significance correlation $(\alpha=.81, p<.01)$. This provides support for the temporal validation of the translated instrument.

Construct Validity: Construct validity of Advance Warning of Relapse (AWARE) Questionnaire-Urdu was established by exploratory factor analysis (EFA) and confirmatory factor analysis (CFA). EFA was used to determine factor structure of AWAREUrdu. Bartlett's test of sphericity was significant $\left(X^{2}(378)=\right.$ $1745.80, p=.000$ ) which demonstrates that the items possess adequate common variance for the conduction of factor analysis; that is, items could lead to the definite factor structures by the exploratory factor analysis (EFA). Kaiser-Meyer-Olkin Measure (KMO) of sample adequacy was .83 which is close to 1 thus indicating that the data set is adequate for the factor structuring ${ }^{28}$.

The factor loadings; that is, how much each factor is contributing towards the construct, indicated that Item-8, 14, 20, and 26 possess poor factor loadings $(\lambda<.30)$. These items are thus removed and EFA was conducted again. Bartlett's test of sphericity was found to be significant $\left(X^{2}(276)=1480.924, p=\right.$ $.000)$ and Kaiser-Meyer-Olkin Measure (KMO) of sample adequacy was also improved to .87. The factor extraction method of Principal 
component analysis (PCA) was used for factor analysis as used by the authors of original version of the AWARE Questionnaire. ${ }^{26}$ Factor analysis results into a unifactorial structure. The analysis demonstrated that 24 retained items of AWARE-Urdu possess good factor loadings $>.30$ and are explaining the $34.3 \%$ of the total variance (Table 2).

The construct validity of this factor structure was established through Confirmatory factor analysis (CFA) by using AMOS 21. This was done by determining the indices of goodness fit for all the items that constitute the AWARE-Urdu so as to validate those items for our sample of study. Results indicate that the model possesses good indices for the fitness (Model-1) which demonstrate that the model is good fit for our data with error covariances applied to decrease error variance and increase the relative true variance. Model-2 however show the model fit without error covariances thus showing poor model fit (Table 3). The model is also shown in Figure 1.

Reliability: The reliability estimates for the Advance Warning of Relapse (AWARE) Questionnaire-Urdu were also estimated which indicated the existence of significantly high reliability $(\alpha=.90)$.

\begin{tabular}{|c|c|c|c|c|c|c|c|c|}
\hline \multirow[t]{2}{*}{ Model } & \multirow[t]{2}{*}{$x^{2}$} & \multirow[t]{2}{*}{ Df } & \multirow[t]{2}{*}{$\boldsymbol{P}$} & \multirow[t]{2}{*}{ CMIN/df } & \multicolumn{4}{|c|}{ Fit indices } \\
\hline & & & & & CFI & NFI & TLI & RMSEA \\
\hline \multicolumn{9}{|c|}{ Model-1 Second Order CFA (24 items - with error covariances) } \\
\hline & 408.93 & 212 & .00 & 1.93 & .92 & .85 & .90 & .06 \\
\hline \multicolumn{9}{|c|}{ Model-2 Second Order CFA (24 items - without error covariances) } \\
\hline & 805.18 & 252 & .00 & 3.19 & .72 & .64 & .69 & .10 \\
\hline
\end{tabular}

Square Error of Approximation

\section{DISCUSSION}

The topic of relapse among the drug addicts is of due importance and has been studied in the past ${ }^{23,29}$. Due to the importance of this construct it is a vital need to measure it across cultures. This purpose could be achieved by translating the instrument measuring relapse prevention in to the culturally relevant language. In the current study the Advance Warning of Relapse (AWARE) Questionnaire ${ }^{26}$ was translated in to Urdu language. This instrument assesses the warning signs of relapse whose timely diagnosis could help to prevent relapse. ${ }^{30}$ The translated version of this instrument could thus help to address the cultural variability and making the measure conceptually understandable as well as reliable across the culture ${ }^{24}$.

Forward-Back translation approach was employed to obtain the translated version. ${ }^{27}$ Two items; Item-22 "I think about drinking" and Item-28 "I am drinking out of control" were modified during the translation phase. In these statements the word "drinking" is replaced by "drug use" because the instrument was to be administered on drug addicts for our research whereas originally the instrument was developed and administered on alcoholics (Table 1). The temporal validity of Urdu version was established by determining the test-retest reliability which provides evidence that the translated version of scale give reliable scores over a time lapse.

The construct validity was also established through Exploratory factor analysis (EFA) and Confirmatory factor analysis (CFA). EFA resulted to obtain a unifactorial structure retaining 24 out of 28 items of the original version (Table 2). Item-8 "I become successful in every plan that I make", Item-14 "I have good eating habits", Item-20 "I am able to think clearly", and Item-26 "I do such activities through which I could abstain from addiction" were removed because of possessing poor factor loadings; which indicated that these items are not significantly explaining variance in the warning signs of relapse as measured by AWARE-Urdu. The reason behind this could be the maintenance of social desirability as all the above-mentioned items are negative predictor of relapse. Literature also supports the fact that drug addicts have a strong desire to maintain socially desirable character and thus exhibit response editing, which is under or over response rate due to the stigmatization being associated with the behavior of addiction ${ }^{31}$.

The factor structure obtained as the result of EFA was confirmed by using Confirmatory factor analysis (CFA). CFA help express the degree of variation between the empirical and predicted factor analysis structures through the measure of chisquare $\left(x^{2}\right)$ and the good fit indices ${ }^{25}$. The indices of model fit (Table 3) provide the support for the construct validity of AWAREUrdu. The reliability estimate provides the support for the reliable nature of scores on AWARE-Urdu.

\section{CONCLUSION}

Relapse, that is a return to addictive behavior signified by recurrence of past condition that exist before treatment, is observed among majority of the drug addicts. It is thus a topic of great interest to be studied. A translated version would help to obtain information more conveniently from drug addicts, however no Urdu translated version is yest devised. The present study thus aimed to translate and validate the instrument for assessing relapse among drug addicts; that is, Advance Warning of Relapse (AWARE) Questionnaire. Findings demonstrate the valid and reliable nature of AWARE-Urdu. The validated Urdu version of AWARE can be used by the researchers to study relapse in drug addicts with understanding of Urdu language. There are also certain limitations; such as the study lacked women sample and comprised only of male drug addicts. The variable sample using random sampling from all the provinces could help in generalization. The cross-language validity for the scale was not established due to unavailability of bilingual sample of drug addicts.

Conflict of interest: Nil

\section{REFERENCES}

1. Auriacombe M, Serre F, Denis C, Fatseas M. Diagnosis of addictions. In: The Routledge handbook of the philosophy and science of addiction, New York: Routledge, 2018;pp 132-144.

2. National Institute of Drug Abuse (NIDA). Understanding Drug Use and Addiction [online] 2018 [cited 2019 Apr 13]. Available from: https://www.drugabuse.gov/publications/drug facts/understandingdrug-use-addiction

3. Levy S, Campbell MD, Shea CL, DuPont R. Trends in abstaining from substance use in adolescents: 1975-2014. Pediatrics 2018;142(2):e20173498. doi:10.1542/peds.2017-3498

4. El-Bassel N, Shaw SA, Dasgupta A, Strathdee SA. Drug use as a driver of HIV risks: re-emerging and emerging issues. Current Opinion in HIV and AIDS. 2014; 9(2):150. doi: $10.1097 / \mathrm{COH} .0000000000000035$

5. Klevens RM, Hu DJ, Jiles R, Holmberg SD. Evolving epidemiology of hepatitis $C$ virus in the United States. Clin Infect Dis 2012;55(suppl_1):S3-9. doi:10.1093/cid/cis393

6. Volkow ND, Baler RD, Compton WM, Weiss SR. Adverse health effects of marijuana use. New Eng J Med 2014;370(23):2219-27. doi:10.1056/NEJMra1402309

7. García-Vita MM, Añaños-Bedriñana FT, Fernández-Sánchez MP. Emotional well-being in women in Spanish prisons: the relevance of the addictive history and the country of origin. Spanish J Prison Health. 2017;19(3):79-86. doi:10.4321/S1575-06202017000300003

8. Daley DC. Family and social aspects of substance use disorders and treatment. J Food Drug Anal 2013;21(4):S73-6. doi:10.1016/j.jfda.2013.09.038 
9. Volkow ND, Baler RD, Goldstein RZ. Addiction: pulling at the neural threads of social behaviors. Neuron 2011;69(4):599-602. doi:10.1016/j.neuron.2011.01.027

10. American Psychiatric Association. American Psychiatric Association: Diagnostic and Statistical Manual of Mental Disorders, Arlington: American Psychiatric Association, 2013; p 81.

11. Gross S. Relapse Prevention [online] 2020 [cited 2019 Apr 28]. Available from: https://psychcentral.com/lib/relapse-prevention/

12. Sinha R, Fox $\mathrm{HC}$, Hong $\mathrm{KI}$, Hansen J, Tuit $\mathrm{K}$, Kreek MJ. Effects of adrenal sensitivity, stress-and cue-induced craving, and anxiety on subsequent alcohol relapse and treatment outcomes. Arch Gen Psych 2011;68(9):942-52. doi:10.1001/archgenpsychiatry.2011.49

13. Azmi AA, Hussin H, Ishak SID, Daud NS (2018). Drug addicts: Psychosocial factor contributing to relapse. In: MATEC Web of Conferences, EDP Sciences, 2018; Vol. 150, p. 05097. doi:10.1051/MATECCONF/201815005097

14. Rahman MM, Rahaman MM, Hamadani JD, Mustafa K, Shariful Islam SM. Psycho-social factors associated with relapse to drug addiction in Bangladesh. J Subst Use 2016;21(6):627-30. doi: 10.3109/14659891.2015.1122099

15. United Nations on Drugs and Crime. Drug use in Pakistan. Ministry of Interior and Narcotics Control Division, Government of Pakistan, 2013.

16. Masood S, Sahar N. An exploratory research on the role of family in youth's drug addiction. J Health Psych Behav Med 2014;2(1):820-32. doi:10.1080/21642850.2014.939088

17. Ogai $Y$, Haraguchi $A$, Kondo A, Ishibashi $Y$, Umeno $M$, Kikumoto $H$, et al. Development and validation of the Stimulant Relapse Risk Scale for drug abusers in Japan. Drug Alcohol Dependence 2007;88(2-3):17481. doi:10.1016/j.drugalcdep.2006.10.005

18. Adinoff B, Talmadge C, Williams MJ, Schreffler E, Jackley PK, Krebaum SR. Time to Relapse Questionnaire (TRQ): a measure of sudden relapse in substance dependence. Am J Drug Alcohol Abuse 2010;36(3):140-9. doi:10.3109/00952991003736363

19. Miller WR, Westerberg VS, Harris RJ, Tonigan JS. What predicts relapse? Prospective testing of antecedent models. Addiction 1996;91(12s1):155-72. doi:10.1046/j.1360-0443.91.12s1.7.x
20. Kelly JF, Hoeppner BB, Urbanoski KA, Slaymaker V. Predicting relapse among young adults: Psychometric validation of the advanced warning of relapse (AWARE) scale. Addict Behav 2011;36(10):987-93. doi:10.1016/j.addbeh.2011.05.013

21. Soundararajan S, Narayanan G, Agrawal A, Murthy P. Personality profile and short-term treatment outcome in patients with alcohol dependence: A study from South India. Indian J Psychol Med 2017;39(2):169. doi:10.4103/0253-7176.203127

22. Gorski T, Miller M. In: Counseling for relapse prevention, Independence, MO: Herald House, 1982.

23. Melemis SM. Focus: Addiction: Relapse Prevention and the Five Rules of Recovery. Yale J Biol Med 2015;88(3):325

24. Huang WY, Wong SH. Cross-cultural validation. In: Encyclopedia of quality of life and well-being research, New York: Springer, 2014; pp. 1369-1371.

25. Prudon $\mathrm{P}$. Confirmatory factor analysis as a tool in research using questionnaires: A critique. Compr Psychol 2015. doi:10.2466/03.CP.4.10

26. Miller WR, Harris RJ. A simple scale of Gorski's warning signs for relapse. J Stud Alcohol 2000;61(5):759-65. doi:10.15288/jsa.2000.61.759

27. Van de Vijver F, Hambleton RK. Translating tests. European Psycho 1996;1(2):89-99. doi: 10.1027/1016-9040.1.2.89

28. Field A. In: Discovering statistics using IBM SPSS statistics, California: Sage Publishers, 2013.

29. Chauhan VS, Nautiyal S, Garg R, Chauhan KS. To identify predictors of relapse in cases of alcohol dependence syndrome in relation to life events. Ind Psychiatry J. 2018;27(1):73. doi: 10.4103/ipj.ipj_27_18

30. Gorski TT. The Cenaps model of relapse prevention: Basic principles and procedures. J Psychoact Drugs 1990;22(2):125-33. doi: 10.1080/02791072.1990.10472538.

31. Krumpal I. Determinants of social desirability bias in sensitive surveys A literature review. Quality \& Quantity 2013;47(4):2025-47. doi: $10.1007 / \mathrm{s} 11135-011-9640-9$ 